\title{
La salud bucal en el marco de \\ la crisis venezolana
}

\section{Oral health in the framework of the Venezuelan crisis}




\section{Introducción}

La caries dental y enfermedad periodontal, son las principales enfermedades que conforman el perfil epidemiológico para el componente bucal de la salud, en buena parte de la población planetaria. A pesar de que dichas enfermedades se pueden prevenir en gran medida, persisten con una alta prevalencia, lo que refleja desigualdades sociales y económicas generalizadas y políticas inadecuadas para la prevención y el tratamiento, particularmente en países con economías dependientes, por lo que son consideradas como un problema mundial de salud pública El modelo médico hegemónico $(\mathrm{MMH})$ en salud que sirve de fundamento conceptual a las políticas en salud bucal, centrado en la alta tecnología y en la atención clínica individualizada, ha sido ineficaz, en el propósito de lograr los cambios estructurales que requiere la población ${ }^{1,2}$.

Para los países latinoamericanos y en general para todos aquellos con economías dependientes, el MMH resulta altamente costoso, excluyente e inequitativo, entre otras razones por la alta dependencia tecnológica, las limitaciones presupuestarias para la atención en salud y la deuda social acumulada que padece buena parte de la población. Desde el Movimiento de la Medicina Social, la Salud Colectiva y la Epidemiología Critica en Latinoamérica se han producido serios cuestionamientos a dicho modelo, así como también todo un desarrollo teórico y metodológico para avanzar en la consolidación de modelos alternativos que permitan superar las limitaciones y el reduccionismo que caracteriza los enfoques tradicionales para la atención en salud. Por su parte la Organización Panamericana de la Salud (OPS), en septiembre de 2014, en ocasión de celebrarse el $53^{\circ}$ Consejo Directivo, aprobó la estrategia para el acceso y la cobertura universal a la salud, enmarcada en los valores y principios de la atención primaria de salud, en el espíritu de Alma-Ata ${ }^{3}$, sin embargo, dichas propuestas han tenido un exiguo impacto en la comunidad odontológica latinoamericana.

Durante los últimos años han surgido voces críticas desde Europa, denunciando el descuido sobre la salud bucal, así como las deficiencias en los sistemas de atención y los servicios preventivos a nivel mundial ${ }^{1}$. En cuanto a las enfermedades bucales se afirma que son un tema desatendido y rara vez visto como una prioridad en las políticas de salud ${ }^{4}$.

En Venezuela la situación del componente bucal de la salud, presenta rasgos excepcionales que ameritan un análisis particular, por la grave crisis que enfrenta la nación como resultado de políticas económicas y sociales inadecuadas $^{5}$. Por una parte, el gobierno ha conformado un marco constitucional y una retórica discursiva populista que declara como fin último de sus acciones, haber elevado la calidad de vida y el bienestar por medio de un conjunto de políticas sociales. El acceso gratuito y equitativo a los servicios de salud, educación, empleo, vivienda, entre otros, son algunos de los logros que intenta exhibir el gobierno en Venezuela, bajo el rótulo de revolución bolivariana sustentada en lo que ellos han denominado como socialismo del siglo XXI. Sin embargo, diversos informes de organizaciones no gubernamentales nacionales e internaciones, reportan las graves condiciones de vida y de salud en que se encuentra la población venezolana. El informe elaborado por Human Rights Watch, el Centro de Salud Humanitaria y el Centro de Salud Pública y Derechos Humanos de la Escuela de Salud Pública Johns Hopkins Bloombereg para la Organización de Naciones Unidas publicado en abril del 2019, reconoce para el escenario internacional que Venezuela enfrenta una emergencia humanitaria compleja y que las políticas sociales implementadas por el gobierno han tenido un exiguo impacto en la población ${ }^{6}$.

La tarea de construir conocimiento sobre la realidad venezolana durante la última década, desde un posicionamiento científico sobre el tema de la salud, exige la delimitación entre el marco jurídico establecido en la Constitución de la República Bolivariana de Venezuela (CRBV), el discurso oficial contenido en planes nacionales de gobierno, programas y normas; y las acciones que finalmente recaen en las condiciones de vida y salud de la población, enfrentando entre otras limitaciones, la política de secuestro de la información en salud y, en particular, de la información epidemiológica ${ }^{7-9}$.

El propósito de esta nota científica es analizar la situación del componente bucal de la salud en la población venezolana en el marco de la crisis nacional. Para alcanzar dicho objetivo se analiza el perfil epidemiológico para el componente bucal de la salud en el marco del derecho a la salud y de las contradicciones entre el discurso oficial y la aplicación de las políticas públicas que finalmente han conducido a la violación de la salud como un derecho humano fundamental, por parte del gobierno nacional.

\section{El derecho a la salud y la participación social en Venezuela}

A mediados del siglo XX en Venezuela se consolidó un sistema de atención a la salud marcado por una concepción centrada en la atención curativa e individual de la enfermedad, positivista, biologicista y reduccionista 10,11. Características que corresponden con el modelo médico descrito por Menéndez ${ }^{2}$. Dicho sistema se fue conformando de forma fragmentada ${ }^{12,13}$ con la incorporación de centros asistenciales planificados, dirigidos y administrados por diferentes instituciones como el Instituto Venezolano de los Seguros Sociales (IVSS), Sanidad Militar, Instituto de Previsión Social del Ministerio de Educación (IPASME) y las Gobernaciones, además de los centros adscritos al Ministerio de Sanidad y Asistencia Social, actualmente MPPS.

Posteriormente, a finales de la década de los noventa y al igual que en otros países de la región, en Venezuela se impulsó la reforma del sector salud, a partir de las propuestas neoliberales emanadas del Fondo Monetario Internacional y el Banco Interamericano de Desarrollo, con el propósito de reducir la participación del Estado en el financiamiento y conducción de los servicios de salud y su transferencia al sector privado ${ }^{14}$.Con la llegada 
de Chávez a la presidencia de la República, se detiene el proceso privatizador de diferentes servicios públicos entre ellos, los correspondientes al sector salud.

El gobierno inicia un proceso constituyente y como resultado del mismo se aprueba la CRBV. Al analizar el marco constitucional, así como el discurso oficial que enmarca las políticas públicas impulsadas en esta década, se evidencia una voluntad política favorable a los sectores populares y otros sectores discriminados y hacia una visión de igualdad sustancial, expresada en una reivindicación del rol del Estado para la corrección de inequidades ${ }^{15}$.

En el nuevo texto constitucional, se puede identificar una concepción del derecho a la salud, a partir de la cual es posible sentar las bases para un nuevo modelo de atención en salud. En los artículos 83, 84 y 85 destacan:

1. El reconocimiento de la calidad de vida, el bienestar colectivo y el acceso a los servicios como determinantes esenciales de las condiciones de salud de la población,

2. Se establece para todas las personas el derecho a la protección de la salud, así como el deber de participar activamente en su promoción y defensa,

3. Se señala en forma explícita la obligación del Estado de establecer un sistema público nacional de salud, de carácter intersectorial, descentralizado y participativo que establezca como prioridad la promoción de la salud y la prevención de las enfermedades,

4. El artículo 85 expresa que el financiamiento del sistema público nacional de salud es obligación del Estado ${ }^{16}$.

Además de ello, en la CRBV se propone la democracia participativa en sustitución de la democracia representativa, para lo cual fueron incorporados unos 70 artículos que promueven la participación ciudadana en varios aspectos de la vida pública del país, muchos de los cuales hacen alusión directa a la participación popular ${ }^{17}$.

La conceptuación que sustenta este articulado en la CRBV, se ubica en el marco de los postulados impulsados desde los paradigmas científicos de la medicina social latinoamericana, la salud colectiva y la epidemiología crítica ${ }^{18}$, al darle rango constitucional a la distribución del poder en los espacios locales de decisión. Se establece explícitamente la participación de las comunidades en la gestión de los centros de salud, así como la obligación de toda la población en participar en su promoción y defensa. El ejercicio de estos derechos constitucionales, que brindan la posibilidad real de tomar decisiones sobre los asuntos públicos, debería conducir a la profundización en el ejercicio de la democracia, la construcción de un sistema público nacional de salud que rompa con el tradicional $\mathrm{MMH}$, así como, a la superación de los principales problemas que genera la pobreza ${ }^{11}$.

Para normar la participación de la población en los asuntos de interés público en los espacios locales, en abril del 2006 se sancionó la Ley que estableció la creación de los
Consejos Comunales, la cual fue reformada en noviembre de 2009 y sancionada como Ley Orgánica de los Consejos Comunales (LOCC). En la misma se establece el marco jurídico para que el pueblo organizado participe directamente en la gestión de las políticas públicas y desarrolle proyectos orientados a responder a las necesidades y aspiraciones de las comunidades, con el propósito de lograr progresivamente mayores niveles de equidad y justicia social ${ }^{19}$.

En la LOCC se establece un conjunto de comités de trabajo siendo el comité de salud el primero que se señala. Cada comunidad debe partir de un diagnóstico de los principales problemas que les afecta, así como de sus potencialidades para elaborar, ejecutar y evaluar el Plan Comunitario de Desarrollo Integral, el cual debe estar articulado a los planes de desarrollo municipal y estadal y con las líneas generales del Plan Nacional Simón Bolívar.

La concepción subyacente sobre el derecho a la salud en la CRBV, así como, sobre la participación social en los asuntos de interés público (LOCC), han permitido a algunos autores como Laurel ${ }^{14}$, Briggs y Mantini-Briggs ${ }^{18}$ describir a Venezuela como un país en el cual los preceptos de la medicina social fueron convertidos en políticas de Estado, para la transformación del sistema nacional de salud y la consolidación de un modelo de atención a la salud basado en la promoción y la prevención. Sin embargo, la situación en que se encuentra la salud de la población venezolana y las instituciones responsables de la prestación de servicios de salud, dista mucho de ser portadora de dichos postulados, contrario a ello, se puede constatar el incumplimiento sistemático de los derechos consagrados en la CRBV.

Uno de los principales incumplimientos del mandato constitucional, ha sido la omisión por parte del gobierno del Past Presidente Chávez para aprobar la Ley Orgánica de Salud, a pesar de los diferentes proyectos de ley preparados y debatidos por la Asamblea Nacional con ese objetivo ${ }^{7}$, especialmente en el período $2006-2011$ en el que tuvo mayoría absoluta el partido de gobierno en el poder legislativo, razón por la cual el derecho a la salud en Venezuela hasta ahora no cuenta con la reglamentación necesaria para su pleno ejercicio.

De igual forma, el gobierno nacional no ha logrado resolver la fragmentación del sistema nacional de salud y la segmentación financiera de las instituciones, que se profundizaron con las reformas neoliberales de los años ochenta y noventa ${ }^{7}$. La creación de la Misión Barrio Adentro que, en sus inicios contribuyó a mejorar el acceso a los servicios de salud para los grupos sociales más desfavorecidos, ha profundizado mucho más la dispersión de la acción gubernamental convirtiéndolo en un sistema de salud paralelo al MPPS, en el cual se generan estadísticas de salud de acceso restringido ${ }^{7}$ que no son compartidas con la información originada en la red pública tradicional.

La fragmentación del sistema de salud, combinada con la disminución de la capacidad para responder a las 
necesidades de atención que demanda la población, incluidas las funciones básicas de vigilancia epidemiológica y la generación de información de salud, ha afectado la prestación de servicios prioritarios de salud pública ${ }^{13}$.

En forma reiterativa el gobierno venezolano señala que ha instaurado un sistema de salud preventivo y participativo, fundamentado en la Misión Barrio Adentro como su eje articulador, sim embargo, los resultados que reflejan los tradicionales indicadores de prevención en salud referidos a las enfermedades transmisibles, la mortalidad materna, la mortalidad infantil y el embarazo en adolescentes, son indicativos del precario desempeño en el trabajo preventivo de Barrio Adentro como sistema de atención primaria ${ }^{20}$.

En cuanto a las posibilidades de participación de la comunidad en la promoción y defensa de la salud, así como en la planificación de los servicios de salud, durante los últimos 20 años se ha producido un proceso gradual de re-centralización de dichos servicios, con los cuales se habían iniciado procesos descentralizadores en los años 90. En el caso de la Misión Barrio Adentro desde sus inicios ha sido dirigida directamente por la Presidencia de la República ${ }^{20}$. La conducción centralizada de todas las instituciones públicas de salud, anula la posibilidad de toma de decisiones en los espacios locales. Los voceros de salud de los consejos comunales han sido utilizados como personal auxiliar de los médicos, en labores de acompañamiento y en algunos casos recibiendo entrenamiento para suplir la falta de personal de enfermería, desvirtuándose el propósito previsto en la CRBV y la LOCC. De manera que las posibilidades de toma de decisiones desde los espacios locales por parte de las comunidades organizadas, es un derecho constitucional inhabilitado por el gobierno nacional, perdiéndose de esta manera el potencial transformador del $\mathrm{MMH}$.

Otro aspecto sustantivo para la crisis del sector salud, es el desfinanciamiento. A pesar de lo que se expresa en los discursos oficiales, el gasto total en salud con relación al PIB en Venezuela no ha experimentado incrementos sensibles en los últimos veinte ańos. Frente al resto de los países suramericanos Venezuela muestra el menor gasto público. Ante la reducción del gasto público en salud, los hogares venezolanos han tenido que asumir una mayor participación en el financiamiento de los gastos de salud, lo que se conoce como gasto de bolsillo. El gasto que cualquier ciudadano venezolano debe sufragar para poder cubrir sus necesidades de salud en los centros asistenciales públicos se encuentra entre los montos más altos de la región de las Américas y se ubica entre los mayores del mundo ${ }^{10}$, A todo paciente que acude a un centro asistencial público se les pide que proporcionen sus propios suministros médicos básicos, como jeringas y escalpelos, y todos los insumos quirúrgicos, hasta la anestesia general en el caso de las intervenciones quirúrgicas. Igualmente, las familias deben proporcionar las comidas para sus familiares convalecientes durante las estadías en el hospital. La inflación y la falta de disponibilidad de medicamentos ha llevado a la creación de un mercado negro y muchas personas aún no pueden pagar los costos de compra ${ }^{9}$. Todo ello es violatorio del derecho a la salud consagrado en el artículo 85 de la CRBV $y$ es una realidad que el gobierno se niega a reconocer ${ }^{1}$.

\section{La salud bucal en Venezuela}

En Venezuela los estudios epidemiológicos para el componente bucal de la salud se inician en el año 1967 con el Estudio para la Planificación Integral de la Odontología (EPIO) ${ }^{21}$; posteriormente el Proyecto Venezuela ${ }^{22}$ en el año 1987 y más recientemente el Perfil socio-epidemiológico de las etnias venezolanas ${ }^{23}$, cuyos resultados fueron publicados en el año 2008. Todos ellos indican que el perfil epidemiológico para el componente bucal de la salud tiene en Venezuela la misma conformación que para otros países del mundo, siendo la principal patología la caries dental, seguida por la enfermedad periodontal.

Los resultados del EPIO arrojaron un índice de CPO (dientes cariados, perdidos y obturados) de 3,94 dientes con experiencia de caries en el grupo de 7 a 14 años ${ }^{21}$. Para 1987 el CPO fue de 3,67 para el grupo etario de 12 años ${ }^{22}$. El tercer estudio epidemiológico nacional, realizado en 2008, fue el primero en el país en emplear la metodología de la OMS ${ }^{23}$. En dicho estudio el índice de CPO para el grupo etario de 5 a 12 años se ubicó en 0,49 y de 2,24 para el grupo etario de 13 a 18 años, lo que indica un importante descenso en la prevalencia de la caries dental, con relación a los resultados obtenidos en años anteriores. Para la población adulta en edades comprendidas entre los 25 y 75 años el CPO va de 5.81 a 23,66, cifras que resultan de una gravedad significativa, siendo la salud bucal un importante componente de la calidad de vida. Para el total de la población venezolana el índice de CPO obtenido en dicho estudio se ubicó en $6,89^{23}$.

Otro aspecto resaltante en este estudio, es el porcentaje de la población con necesidades protésicas insatisfechas, que para los trabajadores indígenas se ubica en $47,7 \%$, seguido del proletariado informal con $32,83 \%$. mientras que el valor más bajo corresponde a la clase social con más altos ingresos, de la cual solo $20,51 \%$ presentan necesidades protésicas insatisfechas, lo cual evidencia que quienes pueden hacer mayor gasto de bolsillo, son los que tienen acceso a la atención odontológica restaurativa ${ }^{24}$.

En cuanto a las políticas de salud bucal impulsadas por el gobierno nacional, conjuntamente con la Misión Barrio Adentro, se puso en marcha Barrio Adentro Odontológico y para el año 2006 la Misión Sonrisa, que debería encargarse de la rehabilitación protésica de los pacientes edentulos a través de una red de consultorios, clínicas populares y Centros de Diagnóstico Integral, que suman 26 Servicios de Atención Odontológica en todo el territorio nacional, según lo señala la Memoria y Cuenta del Ministerio de Salud publicada en el año $2015^{25,26}$. Sin embargo, actualmente estos servicios no están abiertos para el público en general o no ofrecen la asistencia adecuada, por falta de insumos, escasez de medicamentos y 
productos básicos de salud, la falta de servicios públicos (electricidad y agua), y la emigración de personal ${ }^{6-8,20}$.

En los diferentes informes nacionales e internaciones ${ }^{6-8,20}$ que se han elaborado sobre la crisis venezolana y sus repercusiones en la prestación de los servicios de salud a la población, destaca la falta de información acerca de lo que está ocurriendo con relación a los servicios odontológicos en la red asistencial pública, que es la utilizada por el $85,8 \%$ de la población ${ }^{25}$, así como en los servicios privados que también han sido impactados por la catástrofe nacional ${ }^{\text {? }}$.

\section{Programa Nacional de Salud Bucal}

A comienzos del año 2011, MPPS inició un proceso de revisión y actualización de las normas de funcionamiento de los 18 programas de salud, con énfasis en el Primer Nivel de Atención. Se produjo un primer documento organizado en tres volúmenes, para que fuera discutido desde los servicios de salud responsables de la ejecución de dichos programas.

El volumen I referido a las Bases Conceptuales de la Norma Oficial de los Programas que funcionan en la Dirección General de Programas de Salud, del Ministerio del Poder Popular para la Salud. El volumen II: Parámetros de programación, rendimiento y evaluación y el volumen III: Guía práctica para la Atención en el $1^{\circ}$ Nivel de Atención ${ }^{27}$. A efectos de la elaboración de esta investigación, no fue posible tener información alguna referida a los volúmenes II y III, razón por la cual el presente análisis se circunscribe a lo establecido en el volumen I.

Según el MPPS los centros asistenciales públicos se dividen en tres niveles de atención:

- Primer nivel: integrado por Consultorios Populares de Barrio Adentro, Ambulatorios Rurales Tipo I y II y los Ambulatorios Urbanos Tipo I.

- Segundo nivel: integrado por Clínicas Populares, Centros De Atención Odontológica Integral, Centros De Diagnóstico Integral (CDI) y los Centros De Rehabilitación Protésica.

- Tercer nivel: Hospitales Generales y Hospitales Especializados ${ }^{27}$.

Los centros asistenciales que integran el primer nivel de atención, son los responsables del contacto con la comunidad usuaria y serían los encargados de trabajar conjuntamente en acciones de promoción y prevención, además de constituir el eje ordenador de todo el sistema y la fuente primaria de los sistemas de información ${ }^{28}$.

Las funciones y actividades que el Programa Nacional de Salud Bucal (PNSB) prevé para el primer nivel de atención se fundamentan en la elaboración de un plan anual de trabajo a partir del diagnóstico de la situación de salud del área de influencia del servicio, del cual se deben derivar las acciones de promoción y prevención con la participación corresponsable de la comunidad. Además de ello deben cumplir con las actividades de ejecución, control y seguimiento de la atención clínica con tratamientos preventivos, curativos y rehabilitadores.

La política centralizadora que ha venido implementando del gobierno nacional, resulta contraria a lo previsto en el PNSB, ya que no permite que las comunidades participen en la toma de decisiones en los niveles locales sobre la gestión de los servicios de salud, como lo demuestran los indicadores operacionales (Tabla 1), los registros diarios, los formatos DSP04 y el formato para el Consolidado de Actividades Mensuales empleados por el MPPS para todos los servicios odontológicos a nivel nacional ${ }^{31}$, que deben ser elaborados por todos los servicios odontológicos adscritos al MPPS independientemente del nivel de atención (Tabla 2).

Es decir, en cuanto a los resultados de la política de salud que el gobierno se esfuerza en difundir fundamentada en la promoción de la salud y la participación social, no existen evidencias de la efectividad de la promoción de la salud bucal y a los resultados obtenidos según los objetivos propuestos en el PNSB para el primer nivel de atención. No se han incorporado nuevos indicadores que permitan medir el impacto de la participación protagónica de la población en la cogestión de la salud pública, en la formulación de programas y proyectos para la promoción de la salud con las comunidades, así como la participación de los comités de salud en las actividades previstas en el PNSB de acuerdo a lo establecido en la CRBV y en la LOCC para los servicios pertenecientes al primer nivel de atención, incluyendo a los servicios la Misión Barrio Adentro Odontológico ${ }^{31}$. Los indicadores empleados hasta ahora para evaluar a nivel nacional el impacto del PNSB en los servicios odontológicos de la red asistencial pública, se corresponden con la tradicional práctica odontológica curativa e individual propia del MMH (Tablas 1 y 2).

De igual manera, en el documento de la Memoria y Cuenta del MPPS del 2015 entregada a la Asamblea Nacional, las actividades que se reportan como resultados de la política de salud continúan estando circunscritos a la epidemiología tradicional y a los fundamentos del $\mathrm{MMH}$, a pesar de que el discurso oficial pretende posicionar el sistema público de salud en el marco de la medicina social y la salud colectiva ${ }^{30}$.

\section{Nuevas complicaciones epidemiológicas para la salud bucal en Venezuela}

La crisis que atraviesa Venezuela ha afectado directamente el poder adquisitivo de la familia venezolana y en consecuencia sus posibilidades de cubrir las necesidades básicas, como es el caso de la nutrición. Para el año 2017 el $87 \%$ de los venezolanos vive en condiciones de pobreza por ingreso. El $64 \%$ de los venezolanos ha perdido unos 11 kilos de peso entre 2006 y 2017 por el acelerado deterioro de la ingesta de alimentos en la cantidad y calidad necesaria, siendo más afectados los niños y las mujeres. La calidad de la dieta ha empeorado significativamente. En julio de 2018, la desnutrición aguda global en menores de 5 años de las parroquias más pobres del país, tomando en cuenta todos los tipos de desnutrición, 
Tabla 1. Indicadores operacionales de los servicios odontológicos para medir los resultados de la prestación de servicios

\begin{tabular}{|c|c|c|}
\hline $\mathrm{N}^{\circ}$ & \multicolumn{2}{|l|}{ Indicador } \\
\hline 1 & \multicolumn{2}{|l|}{ Instrumento programado } \\
\hline & \multicolumn{2}{|c|}{ IP $=$ Total de horas $\times \mathrm{N}^{\circ}$ de dias hábiles $\times \mathrm{N}^{\circ}$ de odontólogos. } \\
\hline \multirow[t]{2}{*}{2} & \multirow[t]{2}{*}{ Cálculo de las concentraciones } & Total de consultas \\
\hline & & Primeras consultas \\
\hline \multirow[t]{2}{*}{3} & \multirow{2}{*}{$\begin{array}{l}\text { Consultas de tratamiento } \\
\text { odontológico }\end{array}$} & Tratamientos odontológicos \\
\hline & & Primeras consultas \\
\hline \multirow[t]{2}{*}{4} & \multirow[t]{2}{*}{ Consultad de emergencia } & Consulta de emergencia \\
\hline & & Primeras consultas \\
\hline \multirow[t]{2}{*}{5} & \multirow[t]{2}{*}{ Consulta por obturaciones } & Consultas por obturaciones \\
\hline & & Primeras consultas \\
\hline \multirow[t]{2}{*}{6} & \multirow[t]{2}{*}{ Consulta por endodoncia } & Consulta por endodoncia \\
\hline & & Primeras consultas \\
\hline \multirow[t]{2}{*}{7} & \multirow{2}{*}{$\begin{array}{l}\text { Rendimiento abarcado por } \\
\text { consulta }\end{array}$} & Total de consultas \\
\hline & & Instrumento programado \\
\hline \multirow[t]{2}{*}{8} & \multirow[t]{2}{*}{ Consultas por alta } & Total de consultas \\
\hline & & Consultas de alta \\
\hline
\end{tabular}

9 Índice de conservación de los dientes permanentes:

$\frac{\mathrm{N}^{\circ} \text { de dientes permanentes obturados }}{\mathrm{N}^{\circ} \text { permanentes + extracción permanentes }} \times 100$

10 Índice de Atricción

$\frac{\text { Primeras consultas- altas }}{\text { Primeras consultas }} \times 100$

11 Consultas de preventiva escolar

$\frac{\mathrm{N}^{\circ} \text { de escolares consultados }}{\text { Matrícula escolar }} \times 100$


Tabla 2. Indicadores empleados para evaluar el funcionamiento del servicio Odontológico para los diferentes niveles de atención

\begin{tabular}{|c|c|c|}
\hline Regiones & Tipo de Centro asistencial & Indicadores \\
\hline \multirow[t]{11}{*}{ Estado Táchira } & Hospital Central de San Cristóbal & 1. Instrumento programado \\
\hline & Hospital Dr. Ernesto Segundo Paolini & 2. Cálculo de las concentraciones \\
\hline & Hosnital General Padre Justo A rias & 3. Consultas de tratamiento odontológico \\
\hline & & 4. Consultas de emergencia \\
\hline & Centro de higiene escolar N 3 & 5. Consulta por obturaciones \\
\hline & Hospital Militar & 6. Consulta por endodoncia \\
\hline & Hospital San Antonio de Táriba & 7. Rendimiento abarcado por consulta \\
\hline & Ambulatório Urbano Tipo I Abejales & 8. Consultas por alta \\
\hline & Hospital General de Táriba & 9. Índice de conservación de los dientes permanentes \\
\hline & Consultorio Popular tipo III Bramón & 10. Índice de atricción \\
\hline & Ambulatório Urbano Tipo I Soboruco & 11. Consultas de preventiva escolar \\
\hline \multirow[t]{12}{*}{ Estado Mérida } & Ambulatorio Urbano III Belén & \\
\hline & IPASME* Estadal & \\
\hline & Consultorio Popular tipo III La Milagrosa & \\
\hline & Ambulatorio Urbano Tipo I Las Acacias & \\
\hline & IPASME* El Vigía & \\
\hline & Ambulatório Urbano Tipo III Ejido & \\
\hline & Ambulatório Urbano Venezuela Tipo III & \\
\hline & Consultorio Popular Tipo III “La Humboldt” & \\
\hline & Ambulatorio Rural Tipo II El Playón & \\
\hline & Ambulatório Urbano Tipo II de Mucurubá & \\
\hline & Ambulatorio Rural Tipo II El Vallecito & \\
\hline & Consultorio Popular Tipo III Fidel Febres Cordero & \\
\hline \multirow[t]{2}{*}{ Estado Lara } & IPSFA $^{* *}$ & \\
\hline & Hospital Dr. “Luis Gómez López “ & \\
\hline \multirow[t]{6}{*}{ Estado Trujillo } & IPASME*Valera & \\
\hline & $\begin{array}{l}\text { Servicio Odontológico Escuela Nacional Bolivariana } \\
\text { Francisco Briceño Araujo }\end{array}$ & \\
\hline & IPASME* Trujillo & \\
\hline & Ambulatorio Tipo II IPASME*Valera & \\
\hline & Ambulatorio Urbano Tipo I La Sabanita & \\
\hline & Ambulatorio José Vicente Scorza & \\
\hline \multirow[t]{5}{*}{ Estado Barinas } & Consultorio Popular el Limoncito & \\
\hline & $\begin{array}{l}\text { Consultorio Popular Tipo III “Dr. Marcos Mora } \\
\text { Figueroa }\end{array}$ & \\
\hline & Hospital General “Dr. Luis Razetti” & \\
\hline & IPASME* Barinas & \\
\hline & Cruz Roja Venezolana & \\
\hline Estado Nueva Esparta & $\begin{array}{l}\text { Ambulatorio Dr. Emilio Delgado. Servicio Odontológi- } \\
\text { co de la Cruz Roja }\end{array}$ & \\
\hline Estado Monagas & Consultorio Popular Tipo II Divino Niño de Jesús & \\
\hline
\end{tabular}

* Instituto de Previsión Social del Ministerio de Educación

** Instituto de Previsión Social de las Fuerzas Armadas 
fue de $65 \%$, el riesgo nutricional fue de un $34 \%$, y con desnutrición moderada y severa grave fue de $13,5 \%{ }^{31}$.

Cualquier alteración del estado nutricional puede afectar el desarrollo de las estructuras bucales si se presenta a edad temprana, como está ocurriendo con la infancia venezolana. Diversas alteraciones biológicas, anatómicas, funcionales y estéticas de las estructuras dentarias y sus tejidos de sostén, pueden estar ya formando parte del perfil epidemiológico de los niños y adolescentes venezolanos, afectando la relación intermaxilar, produciendo apiñamiento dental y diferentes malformaciones dentarias (como hipoplasia del esmalte), así como el incremento de la prevalencia de caries dental y la enfermedad periodontal ${ }^{32}$, lo que significa un retroceso con relación a los avances logrados en la disminución de la prevalencia de caries dental para los nińos y adolescentes que se habían alcanzado en el año 2008.

Otro problema emergente para la salud colectiva relativa al componente bucal, se refiere al abandono de los controles con el especialista de pacientes que estaban recibiendo tratamientos de ortodoncia, a causa del acelerado empobrecimiento de las familias venezolanas ${ }^{33}$, así como el elevado incremento de los costos para la atención a la salud ${ }^{7}$. El problema radica en que dichos pacientes continúan utilizando la aparatología fija ortodóntica realizando maniobras de auto mantenimiento ${ }^{33}$.

Por otra parte, durante los últimos años han aparecido varios reportajes en la prensa nacional y algunos diarios regionales, denunciando lo que se conoce como "ortodoncia callejera"; vendedores informales que se ubican en las calles y avenidas más céntricas de las principales ciudades del país y ofrecen los servicios de colocación de brackets o venta de los implementos para que las personas se los instalen en sus casas ${ }^{34}$, lo que pude originar un nuevo problema de salud pública a mediano y largo plazo si no se introducen los correctivos necesarios.

\section{Grandes retos para la reconstrucción del sistema público de salud}

La catástrofe social que enfrentamos en Venezuela está configurando un nuevo y complejo perfil epidemiológico en salud bucal, para lo cual se hace necesario el desarrollo de estrategias que permitan a los profesionales de la odontología comprometidos con la salud colectiva, dar respuestas eficaces ante las nuevas necesidades de la población.

Se requiere transformar los tradicionales esquemas epistemológicos y metodológicos para producir conocimiento científico en torno a esta nueva realidad, en la cual se hace más evidente la relación de determinación que existe entre las condiciones sociales y materiales de vida con el comportamiento de la salud-enfermedad bucal. En este sentido, las universidades nacionales tienen mucho que aportar, a partir de la formación de profesionales de la salud conscientes de la realidad actual y comprometidos con los cambios que se requieren para la reconstrucción del sistema nacional de salud. Además de ello, se requiere estructurar nuevas líneas de investigación y generar el co- nocimiento científico necesario para orientar las acciones transformadoras que demanda la superación de la crisis. La creación de foros, grupos de discusión nacionales e internacionales para analizar la nueva realidad nacional y trabajar sobre la salud bucal colectiva, forman parte de las tareas que debemos adelantar.

La reorganización del PNSB requiere de un diagnostico objetivo sobre las condiciones de funcionamiento en que se encuentran los servicios odontológicos, identificando las necesidades de personal a consecuencia del éxodo de profesionales que ha ocasionado la crisis. De igual manera se hace necesario diagnosticar la capacidad operativa de los equipos y las deficiencias en el suministro de materiales odontológicos. Toda esta información requiere ser incorporada en los informes de las organizaciones no gubernamentales nacionales e internacionales que están alertando al mundo sobre la realidad venezolana.

La promoción de la salud y la participación social continúan siendo las estrategias centrales para superar las profundas inequidades en salud que enfrentamos los venezolanos, superando la falaz retórica del gobierno actual, y haciendo uso de los aportes conceptuales y operativos que sobre este tema se han producido tanto en el contexto latinoamericano como a nivel mundial.

\section{Conclusiones}

El actual gobierno nacional se esfuerza en relatar una serie de logros que no tienen correspondencia con las evidencias resultantes de sus políticas sociales y de salud. En el sistema público de salud venezolano no se constatan cambios indicativos del desarrollo de un nuevo modelo de atención en salud que privilegie la promoción de la salud, la prevención y la participación social con expresiones directas en las condiciones de salud bucal y en el funcionamiento de los servicios odontológicos. El tradicional MMH continúa siendo el epicentro de la gestión en salud, contraviniendo el espíritu de la CRBV y violando los derechos humanos fundamentales.

Venezuela, que hasta hace corto tiempo estuvo entre los países más prósperos de América del Sur, ha sido conducido a un profundo retroceso en cuanto al desarrollo político, económico y social. Ahora, más allá del reconocimiento colectivo de la crisis en el contexto internacional, se requiere de la identificación de los recursos, las estrategias y el compromiso de todos los actores sociales implicados en la reconstrucción de la nación y la recuperación de los derechos humanos, sociales y políticos. Proceso en el cual la salud se posiciona como una dimensión de las luchas sociales, pero también de encuentro y de trabajo colectivo para la recuperación de la democracia y la justicia social.

\section{Referencias bibliográficas}

1. Beaglehole R, Beaglehole R. Promoting radical action for global oral health: ¿integration or Independence? The Lancet Health [Internet] 2019 Jul [consultado el 08/01/2020] 20;394(10194):196-198. Disponible en: DOI: $10.1016 / S 0140-6736(19) 31610-1$. 
2. Triana R, Álvarez J. Educación médica, modelo hegemónico y atención primaria de salud. Entrevista al doctor Eduardo L. Menéndez. Praxis \& Saber, [Internet] 2017;8(18):271-298. [Consultado el 04-01-2020] Disponible en: https://dx.doi.org/10.19053/22160159. v8.n18.2017.7251

3. Organización Panamericana de la Salud/ Organización Mundial de la Salud. 162 a del Comité Ejecutivo. Washington, D.C., EUA, del 18 al 22 de junio del 2018 Respuesta de la OPS para mantener una agenda eficaz de cooperación técnica en Venezuela y en los estados miembros vecinos. [Consultado el 11/01/2020]. Disponible en: http://iris.paho.org/xmlui/handle/123456789/49487

4. Peres M, Macpherson L, Weyant R, Daly B, Venturelli R, Mathur MR, et al. Oral diseases: a global public health challenge. The Lancet Health [Internet] 2019 [consultado el 08/01/2020] 20;394(10194):249-260. Disponible en: DOI: 10.1016/S0140-6736(19)31146-8.

5. Kornblihtt J, Dachevsky F. Crisis y renta de la tierra petrolera en Venezuela: crítica a la teoría de la Guerra Económica. Cuadernos del Cendes [Internet] 2017 [Consultado el 15/12/2019] 34(94):1-30 Disponible en: https:// www.redalyc.org/articulo.oa? $\mathrm{id}=40353171002$

6. Human Rights Watch, Venezuela's Humanitarian Emergency Large-Scale UN Response Needed to Address Health and Food Crises. [Internet] Abril 2019. [Consultado el 15/12/2019]. Disponible en: https://www. hrw.org/sites/default/files/report_pdf/venezuela0419_ web_0.pdf

7. Carrillo A. Sistema de salud en Venezuela: ¿un paciente sin remedio? Cad. Saúde Pública [Internet]. 2018 [consultado el 25/11/2019]; 34(3): e00058517. Disponible en: http://www.scielo.br/scielo.php?script=sci_arttext\&pid=S0102-311X2018000305003\&lng=en.

8. Díaz J. Carlos Walter Valecillos: El CENDES y la crisis de la salud en Venezuela. Cuadernos del CENDES [Revista en Internet] mayo-agosto 2016 [Consultado el 05 de enero de 2020]; 33(92)105-112 Disponible en: http://saber.ucv.ve/ojs/index.php/rev_cc/article/ view/12794/12492.

9. Page K, Doocy S, Ganteaume F, Castro J, Spiegel P, Beyrer C. Venezuela's public health crisis: a regional emergency The Lancet Health. [Internet] March 11, 2019. [Consultado el 05/01/2020] Disponible en: http://doi. org/10.1016/S0140-6736(19)30344-7

10. Romero Y. La salud bucal en el marco de las políticas públicas en Venezuela. ODOUS. [Internet] 2005 [Consultado el 25/11/2019]; Vol. VI N². Disponible en: http://servicio.bc.uc.edu.ve/odontologia/revista/index.htm

11. Romero Y. La formación médica en Venezuela: ¿profesionales para la atención de la salud o de la enfermedad? Educere, [S.1.], v. 16, n. 54, p. 59-68, jun. 2018. ISSN 1316-4910. Disponible en: <http://epublica.saber.ula. ve/index.php/educere/article/view/12603>. Fecha de acceso: 19 dic. 2019.

12. Villasana, P. Caraballo J. El sistema de salud en Venezuela y sus políticas públicas: aportes para su integración desde la mirada de la salud colectiva. Salud de los trabajadores. [Internet] 2019; 27(1) 51-64. [Consultado el 04 de enero de 2020]. Disponible de: http://servicio.bc.uc. edu.ve/multidisciplinarias/saldetrab/vol27n1/art05.pdf
13. Organización Panamericana de la Salud/ Organización Mundial de la Salud. 162 del Comité Ejecutivo. Washington, D.C., EUA, del 18 al 22 de junio del 2018 Respuesta de la OPS para mantener una agenda eficaz de cooperación técnica en Venezuela y en los estados miembros vecinos. [consultado el 11/01/2020]. Disponible en: http://iris.paho.org/xmlui/handle/123456789/49487

14. Laurell A. Contradicciones en salud: sobre acumulación y legitimidad en los gobiernos neoliberales y sociales de derecho en América Latina. Saúde Debate [Internet]. 2014 Diciembre [consultadoel07/12/2019];38(103):853-871. Disponible en: http://www.scielo.br/scielo.php?script=sci_arttext\&pid=S0103-11042014000400853\&lng=en.

15. González A. La desigualdad en la Revolución Bolivariana. Una década de apuesta por la democratización del poder, la riqueza y la valoración del estatus. Revista Venezolana de Economía y Ciencias Sociales, [Internet] 2008;14(3)175-199. [Consultado el 2019-1212] Disponible en: http://www.redalyc.org/articulo. oa?id=17721699009

16. Asamblea Nacional. Constitución de la República Bolivariana de Venezuela. Gaceta Oficial Extraordinaria №36.860. [consultado el 12-12-2019] Disponible en https://www.oas.org/dil/esp/constitucion_venezuela.pdf

17. Machado J, Participación social y consejos comunales en Venezuela. Revista Venezolana de Economía y Ciencias Sociales [Internet]. [consultado el 13 de diciembre de 2019] 2009;15(1). Disponible en: https://www.redalyc. org/articulo.oa?id=17721678009

18. Briggs C, y Mantini-Briggs C. "Misión barrio adentro": medicina social, movimientos sociales de los pobres y nuevas coaliciones en Venezuela. Salud Colectiva. [Internet] 2007;3(2):159-176. [consultado el 04/01/2020]. Disponible en: https://doi.org/10.18294/sc.2007.135

19. Asamblea Nacional. Ley Orgánica de los Consejos Comunales. Gaceta Oficial No 39335. 2009. [Internet] [consultado el 18 de diciembre de 2019]. Disponible en: http://www.minci.gob.ve/wp-content/uploads/downloads/2012/11/LEY-CONSEJOS-COMUNALES-611-2012-WEB.pdf

20. Aponte C. Misión Barrio Adentro. Atención Fracturada y Salud en Crisis. Transparencia Venezuela. 2018. [Internet] [consultado el 15/11/2019]. Disponible en: https://transparencia.org.ve/wp-content/ uploads/2018/06/2018-Mision-Barrio-Adentro-Version-TV-completa-1.pdf

21. Cova R. Lozada I. Estudio para la Planificación Integral de la Odontología en Venezuela. Área de Salud Oral. Publicación Oficial del Ministerio de Sanidad y Asistencia Social. Departamento de Odontología Sanitaria. Caracas. 1972

22. Méndez C. Mijares A. Estudio Nacional de Crecimiento y Desarrollo Humano de La República de Venezuela: Aspectos Odontológicos. División de Investigaciones Biológicas. Departamento de Odontología. Tomo III. Caracas. Fundacredesa. 1995

23. Morón A. Perfil epidemiológico bucal de las etnias venezolanas. Primer reporte nacional. Ciencia Odontológica [Internet] 2008;5(3):11[consultado el 12 de enero 2020] Disponible en: https://www.redalyc.org/articulo. oa?id=205216674002 
24. Salazar J. El componente bucal del proceso salud-enfermedad en Venezuela desde una perspectiva social. Acta Odontológica Venezolana. [Internet] 2016 [consultado 15/12/2019]; 54(2). Disponible en: https://www.actaodontologica.com/ediciones/2016/2/art-11/\#

25. Ministerio del Poder Popular para la Salud. Memoria y cuenta del MPPS del 2015 entregada a la asamblea nacional. [Internet] 2015. [consultado el 27/12/2019]. Disponible en http://historico.prodavinci.com/2016/03/04/ actualidad/pdf-esta-es-la-memoria-y-cuenta-entregadapor-el-ministerio-de-salud-a-la-asamblea-nacional/

26. Buada G. Salud bucal de niños venezolanos va en acelerado deterioro. Anmistía internacional Venezuela. [Internet] Julio 2018. [consultado el 16-112019]. Disponible en: https://www.amnistia.org/ve/ blog/2018/07/7206/salud-bucal-de-ninos-venezolanos-va-en-acelerado-deterioro

27. Ministerio del Poder Popular para la Salud. Fundamentos de las normas de los programas de salud. [Internet] [consultado el 01 de diciembre de 2019]. Disponible en: https:/www.mindbank.info/item/3144

28. Almeida G, Artaza O, Donoso N, Fábrega R. La atención primaria de salud en la Región de las Américas a 40 años de la Declaración de Alma-Ata. Rev Panam de Salud Publica [online]. 2018, v. 42 [Accedido 8 enero 2020], e104. Disponible en: <https://doi.org/10.26633/ RPSP.2018.104>.

29. Hernández A. Caracterización de la Red Ambulatoria Convencional Odontológica y la misión Barrio Adentro Odontológica en el Estado Barinas. [Tesis de maestría] [Caracas] Facultad de Odontología. Universidad Central de Venezuela. 2009. 134p [Internet] [consultado el 13/12/2019]. Disponible en: http://hdl.handle. net/123456789/5935
30. Ministerio del Poder Popular para la Salud. Memoria y cuenta del MPPS del 2015 entregada a la asamblea nacional. [Internet] 2015. [consultado el 27/12/2019]. Disponible en http://historico.prodavinci.com/2016/03/04/ actualidad/pdf-esta-es-la-memoria-y-cuenta-entregadapor-el-ministerio-de-salud-a-la-asamblea-nacional/

31. Herrera M. Amenazas al bienestar de la infancia venezolana: Un reto para el pediatra ante una emergencia humanitaria compleja. An Venez Nut. [Internet] 2018 [consultado el 12/01/2020]; 31(2) Disponible en: http://www.analesdenutricion.org.ve/ ediciones/2018/2/art-3/

32. Torres L, Duque J, Granada J, Serna M, García R. Anomalías dentales y su relación con la malnutrición en la primera infancia: un análisis crítico de literatura. Rev Nac Odontol. [Internet]. 30 de junio de 2015 [citado 12 de enero de 2020];11(20). Disponible en: https://revistas.ucc.edu.co/index.php/od/article/view/941

33. Viudch G; Rondón J, Vielma Y, Vielma E; Lindarte N; Loaiza A. et al. Percepción sobre el uso de la ortodoncia correctiva en jóvenes desertores del tratamiento en Mérida. Venezuela. Rev Venez Invest Odont IADR. [Internet] 2020 [Citado el 05/01/2020] 8(1). Disponible en: http://erevistas.saber.ula.ve/index.php/rvio/article/ view/15630

34. Loaiza R. La ortodoncia callejera es la mejor manera de desdibujar sonrisas. Últimas Noticias. [Internet] 2019. [Consultado el 05/01/2020]. Disponible en: http://www.ultimasnoticias.com.ve/noticias/mas-vida/ la-ortodoncia-callejera-es-la-mejor-manera-de-desdibujar-sonrisas/ 